\title{
Small- and medium-sized enterprises' knowledge management systems as edifiers of new value innovations
}

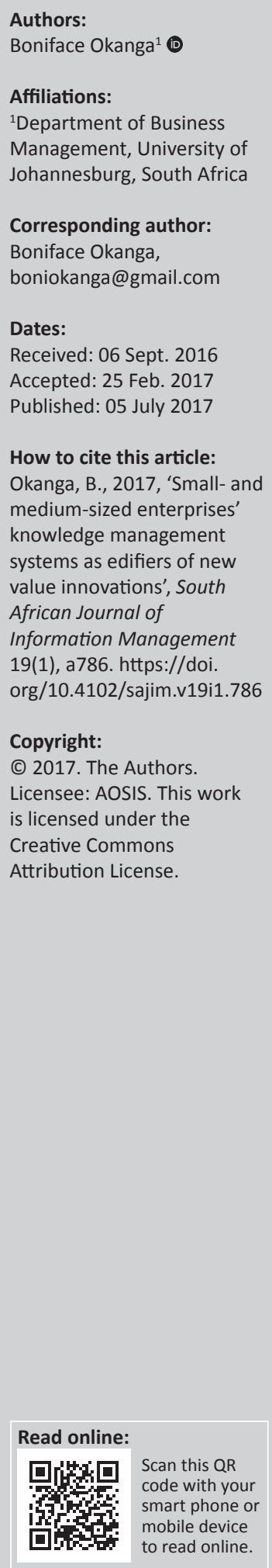

Background: New value innovations are organically ingrained and complementary to the process of knowledge management. However, epistemological trends highlight only limited studies to have explored how the effectiveness of knowledge management leverages new value innovations.

Objectives: This research evaluates how the effectiveness of the small- and medium-sized enterprises' (SMEs) knowledge management systems enhance new value innovations so as to identify the major paradoxes and a business model that coherently integrates knowledge management with new value innovations.

Method: Using a qualitative research method, semi-structured interviews were used to explore the opinions of 20 purposively sampled managers from 20 SMEs on how knowledge management edifies new value innovations.

Results: A strong culture of information utilisation was found to enhance relevant analysis and identification of new gaps in the markets that must be filled through new value innovations or modifications of the existing products. However, poor culture of knowledge utilisation, cost of knowledge management and the often poorly functional business information systems were still reiterated as major paradoxes hampering effective knowledge management as one of the determinants of new value innovations.

Conclusion: The study concluded with a model highlighting a continuum of knowledge management-new value innovation pillars like a strong culture of information utilisation, and encouragement of creativity that the executives must consider to create an effective knowledge management foundation that subsequently leverages the overall effectiveness of new value innovations.

\section{Introduction}

A new value innovation process that is organically intricate, complementary and inherently ingrained with knowledge management leverages the overall effectiveness of new value innovations (Cohendet \& Simon 2015). Effective knowledge management systems enhance thorough analysis and eliciting of sufficient information on the critical unfilled gaps in the markets. This enables the generation of the corresponding array of new ideas on the new product models that must be selected and developed to fill such gaps (Cohendet \& Simon 2015; Zhou \& Li 2012). As firms become more abreast with the existing unmet customer needs and demands and the prevailing nature of the market and industry trends, effective knowledge management also bolsters the executives' ability to effectively deal with the information paradoxes and puzzles on whether new innovations will perfectly match or even exceed customer expectations and perceptions. It also aids the positioning and re-positioning of new innovations in a way that diffuses immediate threats that may emerge from the competitors' retaliations and reactions (Zhou \& Wu 2010).

In other words, a firm's possession of sufficient customer and product information enable necessary reconfigurations and modifications to ensure that the existing product features, attributes and quality are modified to perfectly respond to the changes in customer needs and demands (Zhou \& Li 2012). All these render it glaringly evident that the effectiveness of knowledge management spurs new value innovation and subsequently a business' overall sustainability (Zhou \& Wu 2010). However, a shortfall arises from the fact that most of the studies on new value innovations have often been approached as if innovations are a unique process that does not, in 
any way, relate or get edified by the overall effectiveness of an enterprise's knowledge management system (Hernandez et al. 2010; Leal, Salgueiro \& Ortega 2013; Yayavaram \& Ahuja 2008). Such approach limits the creation of a strong knowledge management culture or the accumulation and management of sufficient information and the tracking of the required unfolding trends to drive the improvement of new value innovations. This study fills this gap by evaluating how the existing small- and medium-sized enterprises' (SMEs) knowledge management systems edify the effectiveness of the process of new value innovations. The motive of the study was to identify the major paradoxes that SMEs face when seeking to utilise their existing knowledge management systems to leverage new value innovations, so as to socially construct a suitable business model that coherently synchronises knowledge management with new value innovations. It is anticipated that the conclusion of this research will motivate most businesses to begin considering the development of an effective knowledge management system as a prerequisite for enhancing the effectiveness of new value innovations.

\section{Literature review}

The argument that effective knowledge management drives new value innovations to leverage a firm's sustainable performance in the increasingly discontinuous contemporary business environment is implicitly echoed in most of the theories on knowledge management and new value innovation (Hernandez et al. 2010; Leal et al. 2013; Yayavaram \& Ahuja 2008).

\section{Knowledge management}

Knowledge management is a strategic process of planning, acquiring, organising and utilising tacit and explicit knowledge and information to create new or modify the existing processes, methods and products. Such initiatives are often undertaken to bolster the improvement of a firm's overall market performance (Nonaka \& Takeuchi 1995; Omotayo \& Olubunmi 2015). It, therefore, edifies not only the improvement of business processes and operational methods, but also constant reconfiguration and modifications to enhance adaptability to the constantly emerging new changes in the external business environment. Although tacit knowledge is the knowledge and ideas possessed by the individual, explicit knowledge is tacit knowledge codified and translated into documents. Nonaka's knowledge creation model posits the interplay between tacit and explicit knowledge that creates new values to often occur through four main interactive modes encompassing socialisation, externalisation, combination and internalisation (Nonaka \& Takeuchi 1995). Socialisation is an interactive process in which the tacit knowledge deeply rooted in the experiences and ideas of different individuals is shared among the participants in a socialisation process (Teo et al. 2011). Although externalisation is the conversion of tacit knowledge into explicit knowledge, combination facilitates the linking and manipulation of different bodies of explicit knowledge to create new knowledge or improve on the existing knowledge. Internalisation is nevertheless the process of generating new tacit knowledge from explicit knowledge (Teng \& Song 2011). However, the effectiveness of knowledge management and the attainment of its associated business values are often predicted by certain eight main steps that include setting the knowledge management goals and knowledge identification, acquisition, development, distribution, use, preservation and measurement (Teng \& Song 2011).

Setting knowledge management goals is often influenced by the results of environmental analysis and forecasting highlighting the prevailing and the likely foreseeable trends vis-à-vis a business' internal capabilities (Bosua \& Venkitachalam 2013). Such analysis highlights the reasons why a particular tacit or explicit knowledge is required to either develop new products or improve the features of the existing products to respond to the unfolding market and industry changes and trends (Bosua \& Venkitachalam 2013). The clear delineation of the essence of knowledge generation influences the identification of the tacit and explicit knowledge that must be acquired. Although explicit knowledge and tacit knowledge can be generated using the available human resources and technologies, it may also in certain cases require recruiting new staffs with requisite skills, competencies and experiences (Bosua \& Venkitachalam 2013). It may also require undertaking investment in relevant technologies and the establishment of the required structures, such as a department for research and innovation.

The assembling of such key capabilities and competencies enhances the development of knowledge and its translation into new values or products (Wang \& Noe 2010). These can contribute enormously to turning around the performance of an organisation. To enable further modifications of the newly developed knowledge, its distribution and sharing through certain defined networks are a prerequisite for it to suit the required needs when it is being used (Wang \& Noe 2010). Although knowledge use can be rendered less important with the passage of time, it is often required that it is well preserved to facilitate easier retrieval in case it is required in the future. Such initiatives must also be accompanied by constant knowledge management to assess the compatibility of the culture that has evolved, and its effects on operational efficiency and the improvement of a business' performance. However, in an argument that seems a modification of Nonaka and Takeuchi's (1995) view of knowledge management, Hedlund and Nonaka (1993) pointed that how an organisation manages its knowledge agents such as individuals, groups, the organisation and inter-organisational agents encompassing customers, suppliers, competitors and other stakeholders determines how it is able to effectively create, transfer and exploit its knowledge resources. As Skandia's Model notes, knowledge management streamlines the flow and management of information to provide the intellectual capital that in turn drives the improvement of an organisation's new innovation initiatives.

\section{Value innovation}

Value innovation is a strategic process of generating relevant information and ideas that are converted into new products 
or the initiatives that modify the existing products or service features (Lee, Olson \& Trimi 2012). Value innovation does not only entail the innovation and production of new products, but also the modifications of the existing products and services to improve the overall level of differentiation and responsiveness to the changes in customers' demands and preferences (Lee et al. 2012). In the process of the modifications of the existing products or the production of new ones, value innovation also edifies a firm's cost competitiveness. It is through value innovation that firms are able to minimise wastes and optimise the usually limited business resources to produce as maximum output as possible. From this view, effective knowledge management is implicitly the epicentre of new value innovation initiatives.

However, such a strong nexus between effective knowledge management and value innovation has not been widely explored by most of the contemporary studies on new value innovations (Kono \& Barnes 2010). Such a view suggests enterprises with underdeveloped knowledge management culture may tend to face challenges as compared to the businesses with entrenched knowledge management culture (Kono \& Barnes 2010). A culture of knowledge management edifies the inquisitiveness of the executives and the managers to devise the best way of generating and utilising the obtained information to provide business advantages for the firm (Hernandez et al. 2010). For knowledge management to translate into the values that enhance value innovation, Cohendet and Simon (2015) argue that it must not only be about generating the required insights, but also the management of ideas as a socio-cognitive process that must be organised and managed. In their analysis, Cohendet and Simon (2015) highlight three new perspectives on how to deal with the challenges of creativity and innovation to entail; the management of the ideation process to foster creativity, the tension between the logic of creation and production, and undertaking a disruptive innovation to transform a traditional industry.

In the process of managing ideation to foster creativity, the challenge is often not the creativity to generate as valuable new ideas as possible, but the translation of such ideas into new viable business concepts (Lee \& Olson 2010). The conversion of the generated new ideas into new viable business concepts requires investment of the executives' time and resources. It also requires the development of the appropriate facilities to enhance the efficient identification, actualisation and extraction of the potentially viable new business concepts from an array of equally competing new ideas (Lee \& Olson 2010). Such a process and capabilities depend on the unique capabilities that each business has. It cannot easily be delegated or replicated by other businesses. Firms that are more proficient at converting ideas into unique business concepts may not only derive unique sources of competitiveness, but also experience consistent superior market performance (Lee \& Olson 2010). The effectiveness of such a process of converting new ideas into tangible business concepts is often further determined by two artefacts encompassing codebook and the manifesto that comprise part of a firm's business culture.

Manifesto refers to the general vision and belief on how new ideas must be used to achieve the desired business benefits and values. Just like the manifesto, codebook defines what the business needs to know and do in order to transform the selected idea into a viable business concept that in turn generates other enormous business values once the ideas have been concretised into a new product, service or a process (Boisot 1987). Even if the business manages to extract a lucrative business concept from the generated ideas, a challenge often still arises from the management of the tension between the logic of creation and the logic of production (Zhou \& Li 2012). The diffusion of such tension is often accomplished using stage-gate process in which after the irreversible process of idea generation, selection and discarding of the unwanted ideas, the process of innovation tend to flow along six other gates that include conception, prototyping, demonstration, production, marketing and testing (Zhou \& Li 2012). It is the complete knowledge of the product in a particular stage that enhances its progress from one stage to the next.

If certain information is not understood or not yet known about a particular product, the product often does not progress to the next stage until all which is required is put in place. An attempt to proceed to the next stage without sufficient information can put the process of innovation and production at a risk of choosing a wrong, yet an irreversible path. The selection of a wrong path can cause waste and costly corrections (Zhou \& Li 2012). Considering the constantly changing market trends and industry turbulence, no new innovations are, however, irresistible to the impetus of change. The emergence of new products, better innovations and other threats implies that constant new ideas must be generated and converted to enable the modifications of the existing products to resist and reshape the emerging new volatile trends to a firm's advantages (Hernandez et al. 2010). In other words, the idea that the effectiveness of knowledge management influences acquisition of extensive knowledge that subsequently spawns the improvement of employee creativity is also echoed in Naggar's (2015) argument in the creativity canvass.

It is argued in the creativity canvass that the effectiveness of the process of new value innovations is not only defined by the extent to which they are perfectively responsive to customer needs and demands. Instead, it is bolstered by the modalities adopted for optimising the existing resources and networks of innovation communities to coherently aid in the identification, building and nurturing of the selected ideas and assets into new value creating new business concepts. However, the knowledge management-related challenges that often still affect such a process are linked to the unwillingness to share ideas and knowledge beyond a trusted network or fear of exposure to criticisms and competition (Zhou \& Li 2012). Besides the poor motivation of the talented employees to generate and share the required critical knowledge and ideas, 
the other challenges are also often associated with lack of the required resources and equipment to facilitate the sharing and improvement of the identified new ideas into new viable business concepts (Zhou \& Li 2012). In other words, theories imply that in new value innovations, knowledge management systems provide sufficient information that leverages creativity, and the generation and conversion of new ideas into new values or viable business concepts. This creates a strong foundation of knowledge management that influences planning, acquisition, organising and utilisation of tacit and explicit knowledge to create new values or improve the existing processes, methods and products to influence a business' overall effective market performance (Hernandez et al. 2010; Leal et al. 2013; Yayavaram \& Ahuja 2008). It enhances ideation, conception, prototyping, demonstration, production, marketing and testing (Cohendet \& Simon 2015:3). All these may impact positively on the improvement of a business sustainability in the increasingly discontinuous business environment (Cunningham 2012; Manzini 2015; Mohsam \& Van Brakel 2011).

As much as it is quite certain that knowledge management spawns the eliciting of relevant information for new value innovations, limited research in that area seems to have affected the emergence of the appropriate business model that the SMEs can emulate to develop more effective knowledge management systems as foundations for new value innovations. This affects the extent to which most of the SMEs are able to continuously undertake new innovations to leverage their sustainability in the increasingly discontinuous contemporary global markets. Since Nonaka's knowledge creation model and Naggar's (2015) creativity canvass have been widely replicated by most of the big businesses, they would offer the most suitable models for SMEs to replicate when seeking to synchronise the effectiveness of knowledge management with the critical processes of new value innovations. However, just like Nonaka's knowledge creation model that limits the evaluation of the interplay between tacit and explicit knowledge to create new values, Naggar's (2015) creativity canvass also only emphasises the essence of creativity as a strategy for leveraging ideation during the innovations. This implies, although Nonaka's knowledge creation and Naggar's (2015) creativity canvass have been widely replicated by most of the big businesses, it may still not be easily practicable for SMEs to easily replicate how effective knowledge management flows to influence not only ideations, but also later modifications and reconfigurations of the product after its introduction into the market. In other words, considering the unique conditions and circumstances in which most SMEs operate, it is such a gap that this research fills by exploring a unique business model that the SMEs can use to facilitate the synchronisation of knowledge management with the relevant processes of new value innovations to leverage their sustainability.

\section{Research statement}

Poorly developed SMEs' knowledge management systems affect the evolution of a culture of information and knowledge utilisation to edify the generation of adequate information that can be analysed to develop an array of ideas on the probable new product developments or modifications of the existing products that can be undertaken.

\section{Aim of the research}

The aim of this research is to evaluate how effectively the existing SMEs' knowledge management systems edify the effectiveness of the process of new value innovations, so as to identify the major inhibitors and a suitable business model that can be extracted to facilitate coherent synchronisation of knowledge management with new value innovations. In a bid to accomplish this, the study was preoccupied with the evaluation of three fundamental questions that included:

- How do the existing SMEs' knowledge management systems edify new value innovations?

- What conditions determine the effectiveness of the SMEs' knowledge management systems as edifiers of new value innovations?

- Which business model can be developed to coherently link and synchronise the process of knowledge management with new value innovations?

\section{Methodology}

Research on knowledge management has led to the development of key steps and constructs that measure the effectiveness of knowledge management. Likewise, the key steps that edify the effectiveness of new value innovations are also well-developed as a result of detailed elucidations in the previous studies. However, the analysis of the linkage between the overall effectiveness of knowledge management and new value innovations has not been widely examined either in the previous quantitative or the qualitative studies. Lack of such a theory constraints the initiatives of most of the businesses engaged in new value innovations to effectively utilise their knowledge management systems to leverage effective management of new value innovations. To develop a grounding theory that SMEs can emulate, this research uses an interpretivist-qualitative research approach to critically analyse different knowledge management literature vis-à-vis new value innovation theories to extract the relevant knowledge management theory that effectively links knowledge management to new value innovations (Clark 2010; Morse 2010). The results of such analysis were triangulated with the interview findings from 20 purposively sampled managers from 20 SMEs.

Purposive sampling was used in the period between March and August 2016 to facilitate the identification and selection of only the SMEs with well-developed knowledge management systems and significant development of a culture of innovation. To accomplish this, the four criteria used in the purposive sampling process entailed ensuring that (1) the selected SME was not only based in Gauteng but also operational in at least three of the nine South African provinces; (2) the SME must have had a turnover of at least 5 million rands in the past 5 years; (3) from the available media reports and other studies, the SME must have had some form 
of industry leadership for better product innovations and better industry practices, and (4) the selected employee to participate in the study must have been either a manager or any key employee well-informed on matters of information technology (IT) applications and knowledge management and its value on new value innovations. Brief analysis of the existing literature and interviews with key employees were conducted with key employees and managers in each of the selected businesses to ensure that the SME to be selected met all these criteria.

The ascertainment of the business' satisfaction of such requirements led to the selection of about 20 SMEs that emerged mainly from the firms in the hospitality, manufacturing, retail, finance and insurance, construction, and logistic handling and distribution industries. Most of the employees that were interviewed were mainly marketing and IT managers due their perceived familiarity with the linkage between IT and new value innovations as compared with the other employees. Interviews were based on semistructured questionnaires to permit further probing and reprobing to elicit detailed information on how the selected SMEs use the process of knowledge acquisition, storage and management to leverage not only new product innovations, but also improvement of their operational processes and methods.

To accomplish this, one-on-one interviews were used to probe whether companies have effective knowledge management systems in place. If so, how such knowledge management systems have been of value during new value innovations or modifications of the existing products or business concepts? This led to the eliciting of the views of the managers on what they perceive to be the conditions that determine the effectiveness of SMEs' knowledge management systems as edifiers of new value innovations, and the type of the business model that they felt could be developed to coherently link and synchronise the process of knowledge management with new value innovations. The obtained qualitative interview data was thematically analysed and triangulated with the results of the interpretations of the theoretical findings on the linkage between knowledge management and new value innovations. Such analysis enabled the reaching of a logical conclusion on the business model that can be extracted and suggested to coherently link and synchronise the process of knowledge management with new value innovations (see Figure 1). The details of the findings are as presented and discussed in the next sections.

\section{Findings}

In line with the research questions for this article, the findings are presented according to the two sub-headings that include:

- SME's knowledge management system as an edifier of new value innovations.

- Determining conditions: SME's knowledge management system as an edifier of new value innovations.

The details are as follows.

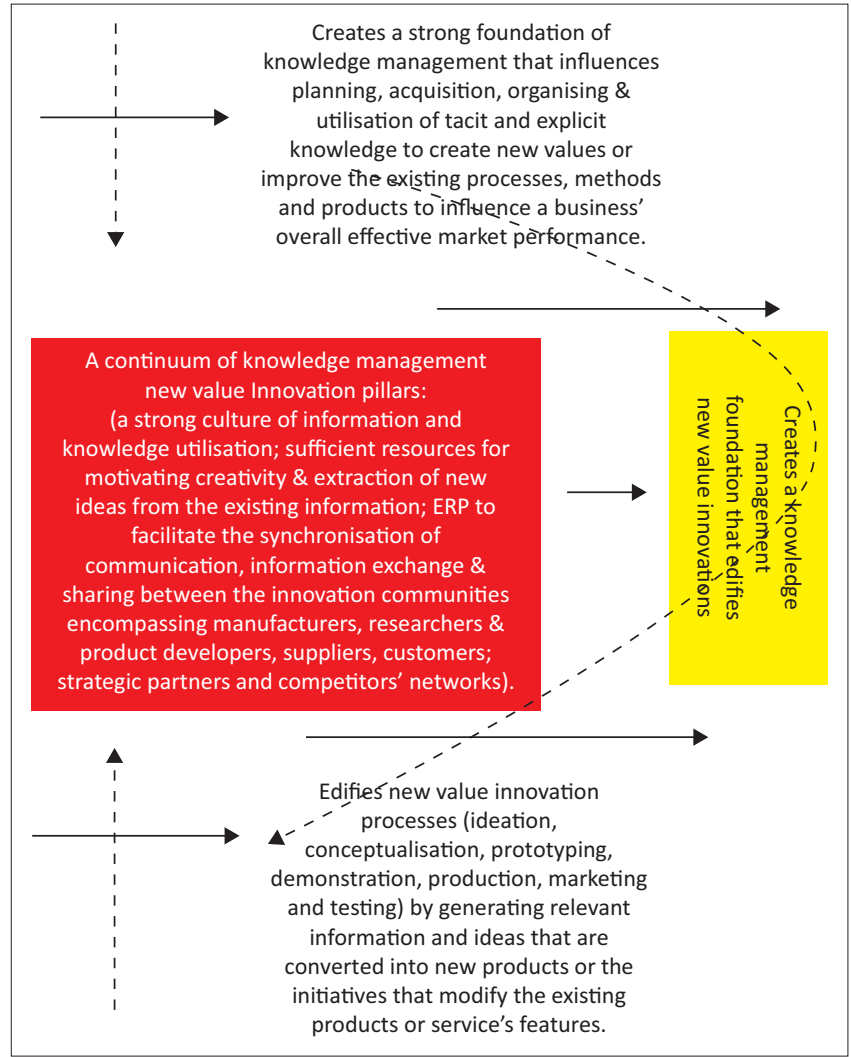

FIGURE 1: A continuum of knowledge management-new value innovation pillars that create an effective knowledge management foundation that edifies the effectiveness of the process of new value innovations.

\section{Small- and medium-sized enterprise's knowledge management system as an edifier of new value innovations}

Thematic analysis of the interview findings indicated the overall effectiveness of most of the SMEs' knowledge management system to leverage new value innovations. In this process, it was found that the effectiveness of SMEs' knowledge management systems does not only provide a foundation for ideation and new innovations, but also reconfigurations, modifications or reshaping of new innovations. The details of these key themes are elucidated as follows.

\section{Provides a foundation for ideation and new innovations}

It emerged from the analysis of the interview data that an entrenched culture of knowledge management provides the foundation for ideation and new product innovations. It facilitates constant analysis and sensing of the probable changes in the industry and market trends. In such analysis, most of the interviewed managers noted that as a firm becomes increasingly inquisitive to gain information and understand the prevailing trends, the enormous amount of the generated information often provides the advantages for the firm to generate different alternatives that must be pursued. This bolsters the creativity of the personnel and the extent to which they are able to emerge with new ideas about the direction that the business must take. It also easily aids the emergence of new ideas for new products that must be introduced to fill the gaps that are 
presently not filled by the business or any other industry operators. In the accomplishment of such practices, some of the managers argued that enterprises that cherish and value information generation and management may, therefore, tend to find it easier to develop new ideas to be narrowed down to the kind of a new business or the product that must be developed. Such a view is accentuated in the opinions of one of the interviewed managers working for a firm in the hospitality industry who stated that:

'For us in the more competitive hospitality industry, new changes and improvements can only be effected if you understand the needs and demands of the customers. For that reason, whether it is a complaint or compliments from the visitors, we usually maintain accurate information of their perceptions of our services such as meals, beddings, cleanliness, safety of the environment and room services. Quite often, we do this directly by conducting a survey or encouraging all our staffs to maintain friendly relationships and to intensely interact with our customers so as to develop conversations that render it possible for us to elicit critical information on how they feel about our services. This enables us to not only understand their perceptions, but also to understand the suggestions on the innovative improvement measures that must be undertaken.' [Participant 16 , female, 33 years, marketing manager]

In other words, when corroborated with the opinions of the other participants, such a findings signifies effectiveness of knowledge management leverages an enterprise's ability to accumulate well documented trends on customer tastes and preferences, the nature of the competition and the degree of industry volatility. It emerged from the interview findings that such analysis also aids the understanding of the supplier and supply chain dynamics and the prediction of the likely dimensions that the existing trends are most likely to undertake in the near future. With such sufficient information, it often turns quite easier for enterprises to narrow down the array of the generated ideas to only a few ideas that are considered to have the potential to turn into viable business ventures or products in the future. In other words, interview findings imply that innovative enterprises with welldeveloped and functional knowledge management systems also tend to possess sufficient knowledge and information, or well-developed techniques for acquiring and storing information that renders it easier for products to be modified and improved through different developmental gates that often encompass conceptualisation, prototyping, development, testing and production. Such a finding is echoed in the views of one of the managers involved in the development and manufacturing of plastic containers in Midrand, who explained that:

'Irrespective of whether we are manufacturing products on orders for certain corporate clients, or we are producing items such as plastic cups, buckets and dishes for the larger markets, my experience indicates that the manufacturing process is a back and forth thing. Initially, research and studies are conducted and the idea that was previously rejected may easily turn useful after the generated prototype has been developed into samples where customers have to provide opinions before undertaking mass production. In that process, you find that although initially what you had imagined and discarded that perhaps the market would not prefer has instead turned into a lucrative item with enormous early trials. That means, you have to go back to the drawing board and redevelop the product to modify the present version to match customer expectations identified during the sale and marketing of the small trial samples. This process goes on like that and of course in our industry, you may develop a particular item and you do not need to develop it again once the market has liked it. However, you still need to expand the product portfolios by conducting new studies as we did recently to venture into the manufacturing of children's plastic toys. That means, you need to logically and systematically keep all the information, product photofits, prototypes and formulas. It saves time for the reason that in case the concept that you previously rejected, turns as the concept that customers prefer, it becomes easier for you to easily retrieve the formulas and information.' [Participant 8, male, 44 years, production manager]

In other words, interview findings signify an effective enterprise knowledge management system that furnishes product developers with enormous critical information that enables them to always make the right decisions. This prevents the likelihood of the process of new product innovations from taking irreversible wrong routes. Sufficient information provided by the enterprise' knowledge management system spawns the improvement of the efficiency of the innovation process. It also aids the elimination of wastes and costly errors that often affect the successful development and launch of new innovations. It is not only during the ideation process that the information generated through an effective knowledge management systems tend to play significant roles, but also after the market launch and introduction of the new product.

\section{Edifies reconfigurations, modifications or reshaping of new innovations}

It emerged from the analysis of the interview findings that one of the causes of the failures of new product innovations is often linked to the use of wrong messages targeted to the wrong segments. Or even lack of complete understanding of the market and industry dynamics in terms of the competitors' responses to the promotional messages upon the new innovations' market introduction. This view is substantiated in the explanations of one of the managers working for a firm engaged in the manufacturing of carpets who stated that:

'Of course, it is true that keeping essential information is important for improving the effectiveness of the process of innovation. However, in practice, there is usually a tendency for more experienced personnel to bypass consultations and exchange of views with customers. In most of the cases, most of the product developers use sketchy information about customer usage and demand for the product to sense and imagine the kind of the product that the customer would prefer. In most of the cases, they score it right just as well as also wrongly. If the prediction and imaginations are wrong, then you loose as the process must be started all over again. But as the new process is being started all over again, information about the reasons why the previous product failed provides critical data that guides decisions on what needs to be done or not to be done to ensure that the new process leads to the development of a concept that 
perfectly matches customer needs. However, that also still depends on whether all the critical information on the failed product was also well stored, preserved and not discarded.' [Participant 11, male, 47 years, chemical engineer]

As some of the managers pointed out, challenges also tend to arise from the failure to gather sufficient information and interpret the likely retaliations or actions that major rivals are most likely to undertake. With the emergence of welldeveloped knowledge management systems, most of the participants reiterated that most of the businesses are often able to deal with such circumstances. Well-developed knowledge management systems do not only provide details on the unfolding market trends, but also critical customer touchpoints or triggers of the interest of certain market segments. Upon the introduction of new innovations, such accurate information provides insights on the critical customer touchpoints that the promotional messages of the new innovations must be based. This enables the undertaking of relevant corresponding actions to improve the positioning of new products in the minds of the targeted market segments, and subsequently the extent of the new innovations' successful launch. Such a finding corroborates the opinions of one of the marketing managers in the tin fish production company who stated that:

'Whether or not the product is successfully launched, our experience indicates that the information that you have in place may also enhance the constant tracking of customer perceptions about the new innovation's market performance. It facilitates the analysis of whether certain customer disappointments are linked to the quality, attributes and the features of the new products, or the pricing and distribution to improve customers' accessibility to new innovations. These enable the enterprise to re-evaluate and undertake the necessary modifications of the quality, features, attributes and distribution approach of the existing products to ensure that they perfectly match or exceed customer perceptions and expectations.' [Participant 14, female, 28 years, sales representative and marketing manager]

In other words, most of the participants seemed to agree that well-developed enterprise knowledge management system also leverages constant analysis and sensing of the probable competitors' reactions upon the introduction of new innovations into the market. It is the understanding of such likely reactions from the competitors that renders it possible for businesses to undertake proactive and precautionary strategic measures to insulate new innovations from any reactions from rivals. If well-developed, managed and utilised, views from most of the managers indicated that the overall effectiveness of an enterprise's knowledge management system can significantly bolster the attainment and sustenance of a firm's superior market performance. However, further analysis of the interview data and triangulation with relevant theories indicated the extent to which an enterprise's knowledge management system is able to edify new value innovations are often predicted by a combination of certain pre-existing conditions (Cant \& Wiid 2013; Olawale \& Garwe 2010; Pillay 2016; Ramukumba 2014; Scheers 2011).

\section{Determining conditions: Small- and medium- sized enterprise's knowledge management system as an edifier of new value innovations}

Further analysis of the interview themes revealed a combination of the pre-existing conditions that often determine whether the SMEs' knowledge management systems are to edify the effectiveness of the process of new value innovations are often linked to a culture of knowledge management and information utilisation, and the availability of information resources and facilities. The details of these themes are evaluated as follows.

\section{Culture of knowledge and information utilisation}

Implicitly, it emerged from the interview data that a culture of knowledge and information utilisation is of significant importance for edifying the effects of knowledge management on new value innovations. A strong culture of knowledge management and information utilisation implies that most of the executives, managers and employees may tend to be motivated to act just on sensing the symptoms of the need to take certain specific actions. It signifies everyone in the organisation must be constantly inquisitive and concerned with how the existing information and new information can be gathered to devise better ways and new unique ideas for improving an enterprise's performance (Naidoo \& Urban 2010). Unfortunately, the interview findings revealed that only a few enterprises tend to exhibit such characteristics. Even for the enterprises with well-developed knowledge management systems, there is often still a challenge of the utilisation of the accumulated information and knowledge to conclude certain critical strategic decisions and actions that can turnaround an enterprise's performance. This is reflected in the views of one of the managers working for a firm involved in the manufacturing of gas from petroleum wastes who stated that:

'From the experience of working across different industries, I have found that some of the executives and most of the employees are often not very bothered on how the existing information or the new ones can be acquired and utilised to develop new ideas and products that can turnaround an enterprise' performance. Instead, businesses tend to work hard to develop one concept or just a few concepts that they utilise for a long time to recoup as much funds used in the development of such a product as possible. During such a process, all new emerging information tend to be ignored. It costs a lot of funds to develop a product, hence, most of the businesses tend to use the existing templates rather than develop new products until it is established that the product's market performance is no longer impressive. In that process, even the staffs that are charged with the tasks of information gathering and analysis tend to only undertake the process of information gathering and analysis just as a daily formality, unless a serious event occurs to force the executives to recognise the importance to recall, analyse and act on the available information.' [Participant 9, male, 43 years, logistics manager]

Even for the businesses that have ended up with certain vibrant business concepts or new innovations, some of the managers reiterated the process of information gathering 
and conceptualisation are often merely based on very sketchy information. The participants attributed this to the fact that some of the executives tend to believe that to emerge with vibrant business concepts, it is not mandatory to have too much information that distorts focus, but only key highlights of major trends. Although such a process often only emerges after the concerned personnel have gained the requisite expertise, it still affects the evolution of a culture of intense information analysis and utilisation among the other staffs. This can place the enterprise at risks by ignoring critical relevant details that should have been considered to ensure that the process of innovation takes a right irreversible path. Whereas poor information and knowledge utilisation are often linked to the deliberate executives' actions, some of the participants highlighted the other challenge that also affects information and knowledge utilisation to create new values to be linked to insufficient resources and facilities.

\section{Information resources and facilities}

To ensure effective utilisation of the existing information to edify the effectiveness of the process of new value innovations, views from most of the managers in the hospitality as well as financial and insurance industries that significantly rely on information systems indicated that investment in the necessary information resources and facilities is a prerequisite. It may require investment in the necessary information systems such as enterprise resource planning (ERP) system. As it emerged from the evaluations of interview data, the establishment of an effective ERP system leverages the improvement of operational efficiency, information gathering, sharing and exchange among the innovation communities that often constitute of the manufacturers, suppliers, customers and the other partner organisations. This is substantiated in the views of one of the managers working with a firm that specialises in the manufacturing and distribution of industrial protective and safety wear who reiterated that:

'Initially, the senior managers ignored the essence for investing in an effective ERP system. However, with time, we found out that giving it a trial would enable us to assess how it would either facilitate or constrain our manufacturing operations. The values turned out to be enormous. Firstly, it facilitated frequent research and analysis to identify areas of safety challenges where we could conduct further research to develop new products to respond to such safety challenges. I mean, there is a lot of information that our research and development departments are able to easily gather not only from interactions with the customers, but also from the other industry players, media houses and suppliers. As the ERP system was developed, it facilitated liaison with the suppliers as well as customers, thereby improving the flow of information along the supply chains. We created a platform and distributed unique identification numbers to all the suppliers and our major customers and distributors. In this process, suppliers just log into our system and assess whether any information has been logged in to suggest that in a week or so we shall run out of the required inputs. Using this information, the suppliers instruct transporters and the logistic service providers to respond by delivering the required materials. As on the other hand, while also using the distributed unique numbers, customers also log in and place the necessary orders that we respond by delivering more effectively. As these services are being used, the query sections tend to provide us with enormous information that we usually analyse to assess how we can not only improve our products or even develop new ones, but also innovate better operational methods and systems.' [Participant 5, female, 26 years, production supervisor]

As ERP system is used to influence the improvement of the efficiency of internal operations as well as acquisitions of more information for further innovations and improvements, some of the managers also reiterated that ERP system is also used to thwart competitive threats in today's volatile competitive landscape. In today's volatile competitive landscape where the use of gaming theory may even entail forging partnerships with some of the rivals in some of the other markets to minimise competition threats, ERP systems are often also extended to create linkages with more friendly competitors or rivals. It is on that basis that ERP improves a firm's rate of the acquisition of new information and the assessment of the probable changes in the market and industry trends. This enables most of the high performing enterprises to identify new trends and respond by either modifying the existing products or developing new products to respond to the unfolding new market needs and demands. As firms enjoy such enormous business values of ERP, it is critical that the executives allocate sufficient resources to ensure that its implementation is a success. Unfortunately, it emerged from the interview data that although most of the enterprises cherish to reap the enormous business values associated with the use of ERP systems, the allocation of the required sufficient financial resources has often been a challenge. Most of the businesses struggle with the issue of sufficient financial resources, with the effect that such a challenge tends to get exacerbated by the hefty costs required for the establishment of an effective ERP system. As for the businesses with sufficient financial resources to establish an effective ERP system, some of the interviewed participants noted that the major constraint has still often arisen from the poor culture of the utilisation of the existing information and new information to develop new products. Such poor culture of information utilisation is often further exacerbated by the inability to read and interpret the accumulated information and knowledge. The inability to read and interpret the accumulated information and knowledge is also in turn affected by insufficient talented staffs to extract new ideas and initiate actions from the information and knowledge that the organisation has at its disposal. This is reflected in the opinions of one of the IT managers working for the manufacturers of industrial chemicals and the other domestic chemicals who stated that:

'Of course the development of an ERP system is critical for influencing the effectiveness of innovation. However, there is usually over excitement at the beginning and after the system is developed, frequent failures arising from poor maintenance tend to affect the use of ERP system in research and innovation. This is because from my experience, we tend to use well developed templates during the daily process of product development. As such templates are being used, frequent improvements and modifications are ignored for fear of deviating from the tested and approved product quality and specifications. This implies 
that even if an ERP system is not functional at some points, it may not matter until it reaches a point where those authorised with product development have identified the need for product improvement or innovation of new products. It is during that process that significant attention is paid towards the improvement of the functionality of the ERP system so as to enhance the understanding of the views of the customers, suppliers, employees and even competitors. Although such a process is critical for aiding the effectiveness of innovation, it is critical to note that during the periods that the system was not functional, a lot of data could have been lost or even the company could have lost track of certain events which could have been of essence for influencing the overall effectiveness of product innovations.' [Participant 19, male, 36 years, manager]

At the same time, it also emerged from the findings that even if the challenge is not insufficient skills, most of the executives are often still quite slow to sense and initiate their own actions from the existing knowledge and information that they have. In most of the cases, there is a tendency to wait and see until the trends that were identified during market and industry analysis have started unfolding. Although the responses are usually reflected in the introduction of new products or modifications of the existing product features and attributes to respond to such new threats, most of the interviewed participants pointed that the failure to utilise the available information to take early actions often cause circumstances, where actions are only undertaken in haste. This affects the importance of calmness and stability that are often the critical prerequisites for undertaking new products' conceptualisation and development. Lack of sufficient financial resources also affects the building of the necessary networks and the motivation of talented employees to continuously search and identify new ideas that can be converted into new viable business concepts.

\section{Discussion}

Survival and sustainability in the increasingly discontinuous business environment require businesses to constantly modify their existing products or develop new products (Cohendet \& Simon 2015). Constant modifications and introduction of new products enhance the extent to which a firm is able to effectively thwart new competitive threats or respond to the changes in the market needs and demands (Cohendet \& Simon 2015). Constant new product development and modifications are also the critical market consolidation or fortifying strategies that enable a firm consolidate their industry positions and thwart risks of infiltration by rivals. However, for firms with less entrenched culture of knowledge management and information utilisation, constant product modifications or innovations may tend to be a challenge (Hernandez et al. 2010; Leal et al. 2013; Yayavaram \& Ahuja 2008). A strong culture of information and knowledge utilisation edifies the accumulation of sufficient information that renders it possible for relevant analysis to be conducted so as to identify new gaps in the markets that must be filled through either new value innovations or modifications of the existing products.
Although this enhances the extent to which the SME's knowledge management system is able to edify new innovation processes, it is also critical that the embracement of a strong culture of information and knowledge utilisation is also accompanied by the dedication of the sufficient financial and non-financial resources (Cant \& Wiid 2013; Naidoo \& Urban 2010; Olawale \& Garwe 2010; Pillay 2016; Ramukumba 2014; Scheers 2011). The allocation of sufficient financial resources can enhance effective use of monetary rewards to motivate talented employees to effectively use the existing information systems to constantly scan the existing information for the unfolding changes and the corresponding new innovations that can be undertaken. This enhances the identification of an array of ideas that can be successfully converted into new viable business concepts or new products. Combined with the establishment of more valuable business information systems such as ERP systems, it is through such approach that the executives can ensure that the improvement in the level of information and knowledge utilisation edify the effectiveness of the entire process of new innovations. In other words, it enhances the improvement of the effectiveness of the process of new innovations that are often linked to ideation, conceptualisation, prototyping, development, market testing, introduction and further modifications to ensure that the new innovations either perfectly match or exceed the customer expectations. However, considering that a culture of information and knowledge utilisation, as well as information resources and facilities were still found to be challenges, it is argued that this research is associated with enormous managerial implications for the contemporary managers.

\section{Managerial implications}

It is indicated in Figure 1 that the managerial implications of the findings signify that managers that aim to undertake constant new value innovations as prerequisites for survival and sustainability in the increasingly discontinuous business environment must consider commencing the process by building a strong culture of knowledge management. This must be undertaken in conjunction with the integration of certain pillars that foster a continuum of knowledge management-new value innovation.

These pillars include a strong culture of knowledge management that emphasises effective information and knowledge utilisation, and sufficient resources for motivating creativity and extraction of new ideas from the existing information. The other pillar would require investment in ERP system to facilitate effective integration and synchronisation of communication, information exchange and sharing among the innovative communities encompassing manufacturers, researchers, product developers, suppliers, customers, strategic partners and competitors' networks. Effective establishment of these pillars creates an effective knowledge management foundation that in turn spawns the effectiveness of the process of new value innovations. This implies that with such a strong culture of information and knowledge utilisation, it becomes easier for enterprises to 
engage in the process of planning, acquiring, organising and utilising tacit and explicit knowledge to create new products or improve the existing processes, methods and products to influence a business' overall effective market performance. It is on that basis that knowledge management also concurrently enhances the generating of relevant information and ideas that are converted into new products or the initiatives that modify the existing products or services' features. In other words, deeper entrenchment of a culture of knowledge management influences the effective accomplishment of critical new value innovation activities that are often linked to ideation, conception, prototyping, demonstration, production, marketing and testing.

\section{Summary and conclusion}

Knowledge management systems provide sufficient information that edifies creativity, and the generation and conversion of new ideas into new values or viable business concepts. This creates a strong foundation of knowledge management that in turn influences planning, acquisition, organising and utilisation of tacit and explicit knowledge to create new or improve the existing processes, methods and products. All these may influence a business' overall effective market performance. Well-developed knowledge management systems do not only provide details on the unfolding market trends, but also critical customer touchpoints or triggers of the interest of certain market segments. Upon the introduction of new innovations, such accurate information provides insights on the critical customer touchpoints that the promotional messages of the new innovations must be based. This enables the undertaking of relevant corresponding actions to improve the positioning of new products in the minds of the targeted market segments, and subsequently the extent of the successful launch of new innovations.

However, as contrasted to the logic in Nonaka's knowledge creation model and Naggar's (2015) creativity canvass that only limited the analysis of the effectiveness of knowledge management as an edifier of the effectiveness of ideation, findings signify knowledge management effectiveness spawns not only the effectiveness of ideation. Instead, it also leverages the entire effectiveness of the process of new value innovations that often spans across ideation, conceptualisation, development, market testing and modifications prior to launching the final product. During the ideation process, the effectiveness of knowledge management enhances the generation of an array of alternative ideas that renders it possible for a firm to select the best ideas. As the conceptualisation, development and market testing processes unfold, knowledge management aids further modifications and reconfigurations to improve the extent to which the product is able to perfectly match or even exceed customer expectations. Although this spurs the development and market introduction of the best concept, quite often, that it is often not the end of all the processes of new value innovations.

Constant changes in market and industry trends may require constant review and modifications of the product to enable it remain consistently relevant to the changes in market needs. This implies that even after the launch of the final product, an entrenched culture of information and knowledge utilisation still leverages the accumulation and management of sufficient information that in turn spurs relevant analysis to identify new gaps in the markets that must be filled through improvements or modifications of the existing products. In other words, the maintenance of an efficiently functional knowledge management system facilitates consistent and systematic analysis and generation of critical information for firms to understand the unfolding trends and to modify the already introduced products to match such trends. It facilitates the analysis of whether certain customer disappointments are linked to the quality, attributes and the features of the new products, or the pricing and distribution to improve the customers' accessibility to new innovations. These enable the enterprise to re-evaluate and undertake the necessary modifications of the quality, features, attributes and distribution approach of the existing products to ensure that they perfectly match or exceed customer perceptions and expectations.

As much as such frequent product modifications improve the strategic fitness between the firm and the unfolding industry and market trends, it is often not only product modifications facilitated by effectiveness of knowledge management that improves a strategic fit between the enterprise and such unfolding market and industry trends, but also the extent of a firm's operational efficiency to effectively match their needs and demands. This implies with an entrenched culture of knowledge management, it often turns easier for firms to conduct relevant analysis to identify and correct deviating costly processes that may affect cost and quality management. It is also during such process analysis and evaluation that firms are able to effectively modify and improve their processes, work methods and operational efficiency to meet the emerging changes in market demands and needs. All these enhance the improvement of the survival and sustainability of the business in the increasingly discontinuous business environment. However, it is still often quite critical that the embracement of a strong culture of information and knowledge utilisation is accompanied by the dedication of sufficient financial and non-financial resources towards the development of effective knowledge management systems.

As the effectiveness of knowledge management facilitates the generation of alternative ideas, the availability of the required financial resources and skills titivates the effectiveness of the implementation of new innovations and improvement measures that must be undertaken. Unfortunately, it emerged from the findings that despite a largely infant level of innovation culture among most of the SMEs, the effective utilisation of their knowledge management systems to leverage the effectiveness of new value innovations is still often undermined by poor maintenance of knowledge management systems. Besides lack of a clear business model linking knowledge management to the effectiveness of new value innovations, the other challenges are often linked to inadequate financial resources, skills and facilities. This study attempted to address such challenges by 
suggesting a continuum of knowledge management-new value innovation pillars (a strong culture of knowledge management that emphasises effective information and knowledge utilisation, and sufficient resources for motivating creativity and extraction of new ideas from the existing information) that the executives must consider to create an effective knowledge management foundation that subsequently edifies the effectiveness of the process of new value innovations. However, future research can still explore how new value innovations can be used as a driver for reshaping the increasingly discontinuous industries and markets.

\section{Acknowledgements Competing interests}

The author declares that he has no financial or personal relationships that may have inappropriately influenced him in writing this article.

\section{References}

Boisot, M., 1987, Information and organisations: The manager as anthropologist Fontana-Collins, London.

Bosua, R. \& Venkitachalam, K., 2013, 'Aligning strategies and processes in knowledge management', Journal of Knowledge Management 17(3), 331-346. https://doi. org/10.1108/JKM-10-2012-0323

Cant, M.C. \& Wiid, J.A., 2013, 'Establishing the challenges affecting South African SMEs', International Business \& Economics Research Journal 12(6), 707-716. https://doi.org/10.19030/iber.v12i6.7869

Clark, V.L., 2010, 'The adoption and practice of mixed methods: US trends in federally funded health-related research', Qualitative Inquiry 16(1), 428-440. https://doi. org/10.1177/1077800410364609

Cohendet, P. \& Simon, L., 2015, 'Introduction to the special issue on creativity in innovation', Technology Innovation Management Review 22(4), 122-204.

Cunningham, S., 2012, The fundamentals of innovation system promotion for development practitioners, Mesopartner, Pretoria.

Hedlund, G. \& Nonaka, I., 1993, Models of knowledge management, Macmillan, London.

Hernandez, M.R., Cepeda, C.G., Cegarra, N.J.G. \& Leal, M.A., 2010, 'The role of cultural barriers in the relationship between open-mindedness and organisational innovation', Journal of Organisational Change Management 23(4), 360-376. https://doi.org/10.1108/09534811011055377

Kono, P.M. \& Barnes, B., 2010, 'The role of finance in the strategic-planning and decision-making process', Graziadio Business School Review 12(1), 19-87.
Leal, M.A., Salgueiro, J.L. \& Ortega, S., 2013, 'Knowledge management and the effectiveness of innovation outcomes: The role of cultural barriers', The Electronic Journal of Knowledge Management 11(1), 62-71.

Lee, S. \& Olson, D., 2010, Convergenomics: Strategic innovation in the convergence era, Gower Publishing, Surrey.

Lee, S., Olson, D. \& Trimi, S., 2012, 'Co-innovation: Convergence, collaboration, and co-creation for organizational values', Management Decision 50(5), 817-831. https://doi.org/10.1108/00251741211227528

Manzini, S.T., 2015, 'Measurement of innovation in South Africa: An analysis of survey metrics and recommendations', South African Journal of Science 11(12), 1-8. https://doi.org/10.17159/sajs.2015/20140163

Mohsam, F. \& Van Brakel, P.A., 2011, 'Information and knowledge sharing trends of small and medium-sized enterprises in the Western Cape, South Africa', South African Journal of Information Management 13(1), 1-10. https://doi.org/10.4102/ sajim.v13i1.462

Morse, J.M., 2010, 'Simultaneous and sequential qualitative mixed method designs', Qualitative Inquiry 16(1), 483-491. https://doi.org/10.1177/1077800410364741

Naggar, R., 2015, 'The creativity canvas: A business model for knowledge and idea management', Technology Innovation Management Review 5(7), 50-58.

Naidoo, R. \& Urban, B., 2010, 'The relevance of operational skills towards business sustainability: A focus on SME manufacturers in the Vaal Triangle region', Acto Commercii 1(1), 234-248.

Nonaka, I. \& Takeuchi, K., 1995, The knowledge creating company: How Japanese companies create the dynamics of innovation? Oxford University Press, London.

Olawale, F. \& Garwe, D., 2010, 'Obstacles to the growth of new SMEs in South Africa: A principal component analysis approach', African Journal of Business Management 1(3), 729-738.

Omotayo, F. \& Olubunmi, M., 2015, 'Knowledge management as an important tool in organisational management: A review of literature', Library Philosophy and Practice 12(8), 1-23.

Pillay, K., 2016, South Africa manufacturing industry leader, Deloitte, Johannesburg.

Ramukumba, T., 2014, 'Overcoming SMEs challenges through critical success factors: A case of SMEs in the Western Cape Province, South Africa', Economic and Business Review 16(1), 19-38.

Scheers, L.V., 2011, 'SMEs marketing skills challenges in South Africa', African Journal of Business Management 4(1), 504-525.

Teng, J.T.C. \& Song, S., 2011, 'An exploratory examination of knowledge-sharing behaviours: Solicited and voluntary', Journal of Knowledge Management 15(1), 104-117. https://doi.org/10.1108/13673271111108729

Teo, T., Nishant, R., Goh, M. \& Agarwal, S., 2011, 'Leveraging collaborative technologies to build a knowledge sharing culture at HP analytics', MIS Quarterly Executive 10(1), 1-18.

Wang, S. \& Noe, R.A., 2010, 'Knowledge sharing: A review and directions for future research', Human Resource Management Review 20(2), 115-131. https://doi. research', Human Resource Ma
org/10.1016/j.hrmr.2009.10.001

Yayavaram, S. \& Ahuja, G., 2008, 'Decomposability in knowledge structures and its impact on the usefulness of inventions and knowledge-based malleability', Administrative Science Quarterly 53(2), 333-362. https://doi.org/10.2189/asqu.53.2.333

Zhou, K.Z. \& Li, C.B., 2012, 'How knowledge affects radical innovation: Knowledge base, market knowledge acquisition, and internal knowledge sharing', Strategic Management Journal 3(1), 219-277. https://doi.org/10.1002/smj.1959

Zhou, K.Z. \& Wu, F., 2010, 'Technological capability, strategic flexibility, and product innovation', Strategic Management Journal 31(5), 547-561. 